La comparaison des différentes fractions azotées dans le muscle peaucier d'animaux de I 8 mois révèle que la concentration de l'azote sarcoplasmique est plus élevée chez l'animal culard $(27,1$ I p. I 00 contre 24,55 p. Ioo de l'azote soluble). L'azote du stroma représente I 3,85 p. Ioo de l'azote total chez les animaux normaux, 8,79 p. Ioo chez les culards. La concentration en myoglobine de ce muscle apparaît équivalente chez les 2 types d'animaux. A l'âge moyen de 42 jours (culards) et de 62 jours (normaux) les rapports GAPDH/EC, GAPDH/HOAD, LDH/EC sont semblables dans le muscle semi-tendineux ou long vaste. Seul le rapport phosphorylase/HK apparaît abaissé chez les culards. Tous ces rapports sont augmentés chez l'animal adulte normal et diminués dans le cceur. On n'a pas trouvé de différences quant au pourcentage de sujets hétérozygotes, pour les loci Mor-I et PMGI, chez les animaux normaux ou culards.

\title{
CONSÉQUENCES DÉ L'HYPERTROPHIE MUSCULAIRE HÉRÉDITAIRE DES BOVINS SUR LA MUSCULATURE \\ I. - IMPORTANCE REIATIVE DES DIFFÉRENTES RÉGIONS
}

R. Boccard et B.-L. Dumont. - Station de Recherches sur la Viande, C. R. Z. V., I. N. R. A., Theix, 63 - Saint-Genès-Champanelle (France). - Laboratoire de Recherches sur la Viande C. N.R. Z., I. N. R. A., 78-Jouy-en-Josas (France).

Les résultats de la dissection complète de la musculature des demi-carcasses de 9 taurillons hypertrophiés et de $\mathbf{I} 7$ taurillons normaux de race charolaise de Io à 20 mois ont été traités pour comparer la part relative des différentes régions musculaires. La comparaison a été effectuée après calcul par régression, pour chaque région, du poids qu'elle représentait dans une masse de $\mathrm{I}_{4} \mathrm{O} \mathrm{kg}$ de muscle dans une demi-carcasse. Les différents muscles ont été regroupés en onze régions dont l'importance relative a été appréciée par le rapport $\frac{\mathrm{H}-\mathrm{N}}{\mathrm{N}} \times$ roo où $\mathrm{H}$ est le poids de la région du type hypertrophié et $\mathrm{N}$ le poids de celle du type normal. Les valeurs obtenues furent pour chaque région : cervicale $-6,3 \mathrm{I}$; antibrachiale $+0,16$; brachiale $+5,02$; scapulaire $-5,72$; rachidienne $-8,46$; thoracique +6.77 ; diaphragmatique - I8,05; abdominale $-5,84$; fessière $-4,44$; crurale $+9,92$ et jambière $-6,44 \mathrm{p}$. Ioo.

Il apparaît donc qu'à poids de muscle constant l'hypertrophie affecte uniquement les régions crurale, thoracique et brachiale et que les autres régions sont retardées dans leur développement à des degrés divers.

Les modifications constatées dans l'importance relative des masses musculaires des différentes régions constituent une exception à la loi d'harmonie anatomique. La différence de répartition entre régions explique, en partie, les différences d'aspect et de conformation qui séparent les deux types d'animaux (finesse de l'encolure, ventre levretté, membre postérieur dit " cul de poulain ") et que l'on peut apprécier sur une base quantitative d'après la valeur du rapport du poids des muscles de la cuisse au poids des muscles du cou $(2,27$ pour les hypertrophiés contre 1,94 pour les normaux) ou encore à celui des muscles de la région abdominale inférieure $\left(3,98\right.$ pour les hypertrophiés contre $3,3^{6}$ pour les normaux).

\section{CONSÉQUENCES DE L'HYPFRTROPHIE MUSCULAIRE HÉRÉDITAIRE DES BOVINS SUR LA MUSCUL,ATURE}

\section{II. - IMPORTANCE RELATIVE DES DIFFÉRENTS MUSCLES}

B.-L. Dumont et R. Boccard. - Laboratoive de Recherches sur la Viande, C. N.R. Z., I. N. R. A., 78 - Jouy-en-Josas (France). - Station de Recherches sur la Viande, C. R. Z. V., I. N.R. A., Theix, 63-Saint-Genès-Champanelle (France).

On a comparé, après calcul par régression des poids qu'ils représenteraient dans une masse de $140 \mathrm{~kg}$ de muscle par demi-carcasse, le poids des principaux muscles de taurillons charolais de Io à 20 mois, de type normal $(n=$ I 7$)$ et de type hypertrophié dit " culard " $(n=9)$. La 